\title{
On Minkowski's inequality and its application
}

\author{
Chang-Jian Zhao ${ }^{{ }^{*}}$ and Wing-Sum Cheung ${ }^{2}$
}

* Correspondence: chjzhao@163. com

${ }^{1}$ Department of Mathematics, China Jiliang University, Hangzhou 310018, China

Full list of author information is available at the end of the article

\section{Abstract}

In the paper, we first give an improvement of Minkowski integral inequality. As an application, we get new Brunn-Minkowski-type inequalities for dual mixed volumes. 2000 Mathematics Subject Classification: 52A30, 52A40, 26D15

Keywords: Minkowski?'?s inequality, H?ö?lder?'?s inequality, Brunn-Minkowski inequality, dual mixed volume

\section{Improvement of Minkowski's inequality}

The well-known inequality due to Minkowski can be stated as follows ([1], pp. 19-20, [2], p. 31]):

Theorem 1.1 Let $f(x), g(x) \geq 0$ and $p>1$, then

$$
\left(\int(f(x)+g(x))^{p} \mathrm{~d} x\right)^{1 / p} \leq\left(\int f(x)^{p} \mathrm{~d} x\right)^{1 / p}+\left(\int g(x)^{p} \mathrm{~d} x\right)^{1 / p}
$$

with equality if and only if $f$ and $g$ are proportional, and if $p<1(p \neq 0)$, then

$$
\left(\int(f(x)+g(x))^{p} \mathrm{~d} x\right)^{1 / p} \geq\left(\int f(x)^{p} \mathrm{~d} x\right)^{1 / p}+\left(\int g(x)^{p} \mathrm{~d} x\right)^{1 / p}
$$

with equality if and only if $f$ and $g$ are proportional. For $p<0$, we assume that $f(x), g$ $(x)>0$.

An (almost) improvement of Minkowski's inequality, for $p \in \mathbb{R} \backslash\{0\}$, is obtained in the following Theorem:

Theorem 1.2 Let $f(x), g(x) \geq 0$ and $p>0$, or $f(x), g(x)>0$ and $p<0$. Let $s, t \in \mathbb{R} \backslash\{0\}$, and $s \neq t$. Then

(i) Let $p, s, t \in \mathbb{R}$ be different, such that $s, t>1$ and $(s-t) /(p-t)>1$. Then

$$
\begin{gathered}
\int(f(x)+g(x))^{p} \mathrm{~d} x \leq\left[\left(\int f^{s}(x) \mathrm{d} x\right)^{1 / s}+\left(\int g^{s}(x) \mathrm{d} x\right)^{1 / s}\right]^{s(p-t) /(s-t)} \\
\times\left[\left(\int f^{t}(x) \mathrm{d} x\right)^{1 / t}+\left(\int g^{t}(x) \mathrm{d} x\right)^{1 / t}\right]^{t(s-p) /(s-t)}
\end{gathered}
$$

with equality if and only if $f(x)$ and $g(x)$ are constant, or $1 / p=(1 / s+1 / t) / 2$ and $f(x)$ and $g(x)$ are proportional.

(ii) Let $p, s, t \in \mathbb{R}$ be different, such that $s, t<1$ and $s, t \neq 0$, and $(s-t) /(p-t)<1$. Then

\section{Springer}

(C) 2011 Zhao and Cheung; licensee Springer. This is an Open Access article distributed under the terms of the Creative Commons Attribution License (http://creativecommons.org/licenses/by/2.0), which permits unrestricted use, distribution, and reproduction in any medium, provided the original work is properly cited. 


$$
\begin{gathered}
\int(f(x)+g(x))^{p} \mathrm{~d} x \geq\left[\left(\int f^{s}(x) \mathrm{d} x\right)^{1 / s}+\left(\int g^{s}(x) \mathrm{d} x\right)^{1 / s}\right]^{s(p-t) /(s-t)} \\
\times\left[\left(\int f^{t}(x) \mathrm{d} x\right)^{1 / t}+\left(\int g^{t}(x) \mathrm{d} x\right)^{1 / t}\right]^{t(s-p) /(s-t)},
\end{gathered}
$$

with equality if and only if $f(x)$ and $g(x)$ are constant, or $1 / p=(1 / s+1 / t) / 2$ and $f(x)$ and $g(x)$ are proportional.

Proof (i) We have $(s-t) /(p-t)>1$, and in view of

$$
\int(f(x)+g(x))^{p} \mathrm{~d} x=\int\left[(f(x)+g(x))^{s}\right]^{(p-t) /(s-t)} \cdot\left[(f(x)+g(x))^{t}\right]^{(s-p) /(s-t)} \mathrm{d} x .
$$

By using Hölder's inequality (see [1] or [2]) with indices $(s-t) /(p-t)$ and $(s-t) /(s-$ $p$ ), we have

$$
\int(f(x)+g(x))^{p} \mathrm{~d} x \leq\left[\int(f(x)+g(x))^{s} \mathrm{~d} x\right]^{(p-t) /(s-t)}\left[\int(f(x)+g(x))^{t} \mathrm{~d} x\right]^{(s-p) /(s-t)},
$$

with equality if and only if $(f(x)+g(x))^{s(p-t) /(s-t)}$ and $(f(x)+g(x))^{t(s-p) /(s-t)}$ are proportional, i.e., either $f(x)+g(x)$ is constant or the exponents are equal, i.e., $1 / p=(1 / s+$ $1 / t) / 2$.

On the other hand, by using Minkowski's inequality for $s>1$ and $t>1$, respectively, we obtain

$$
\left(\int(f(x)+g(x))^{s} \mathrm{~d} x\right)^{1 / s} \leq\left(\int f^{s}(x) \mathrm{d} x\right)^{1 / s}+\left(\int g^{s}(x) \mathrm{d} x\right)^{1 / s},
$$

with equality if and only if $f(x)$ and $g(x)$ are proportional, and

$$
\left(\int(f(x)+g(x))^{t} \mathrm{~d} x\right)^{1 / t} \leq\left(\int f^{t}(x) \mathrm{d} x\right)^{1 / t}+\left(\int g^{t}(x) \mathrm{d} x\right)^{1 / t},
$$

with equality if and only if $f(x)$ and $g(x)$ are proportional.

From (1.5), (1.6) and (1.7), (1.3) easily follows. From the equality conditions of (1.5), (1.6) and (1.7), the case of equality stated in (i) follows.

(ii) We have $(s-t) /(p-t)<1$. Similar to the above proof, we have

$$
\int(f(x)+g(x))^{p} \mathrm{~d} x \geq\left[\int(f(x)+g(x))^{s} \mathrm{~d} x\right]^{(p-t) /(s-t)}\left[\int(f(x)+g(x))^{t} \mathrm{~d} x\right]^{(s-p) /(s-t)},
$$

with equality if and only if either $f(x)+g(x)$ is constant or $1 / p=(1 / s+1 / t) / 2$.

On the other hand, in view of Minkowski's inequality for the cases of $0<s<1$ and 0 $<t<1$,

$$
\left(\int(f(x)+g(x))^{s} \mathrm{~d} x\right)^{1 / s} \geq\left(\int f(x)^{s} \mathrm{~d} x\right)^{1 / s}+\left(\int g(x)^{s} \mathrm{~d} x\right)^{1 / s},
$$

with equality if and only if $f(x)$ and $g(x)$ are proportional, and

$$
\left(\int(f(x)+g(x))^{t} \mathrm{~d} x\right)^{1 / t} \geq\left(\int f(x)^{t} \mathrm{~d} x\right)^{1 / t}+\left(\int g(x)^{t} \mathrm{~d} x\right)^{1 / t},
$$

with equality if and only if $f(x)$ and $g(x)$ are proportional. 
The inequality (1.4) easily follows, with equality as stated in (ii).

Remark 1.3 For (i) of Theorem 1.2, for $p>1$, letting $s=p+\varepsilon, t=p-\varepsilon$, when $p, s, t$ are different, $s, t>1$, and $(s-t) /(p-t) / 2>1$, and letting $\varepsilon \rightarrow 0$, we get (1.1).

For (ii) of Theorem 1.2, for $p<1$ and $p \neq 0, s=p+\varepsilon, t=p+2 \varepsilon$, when $p, s, t$ are different, $s, t<1$ and $s, t \neq 0$, and $(s-t) /(p-t)=1 / 2<1$, and letting $\varepsilon \rightarrow 0$, we get (1.2).

\section{An application}

The setting for this paper is $n$-dimensional Euclidean space $\mathbb{R}^{n}(n>2)$. Associated with a compact subset $K$ of $\mathbb{R}^{n}$, which is star-shaped with respect to the origin, is its radial function $\rho(K, \cdot): S^{n-1} \rightarrow \mathbb{R}$, defined for $u \in S^{n-1}$, by

$$
\rho(K, u)=\operatorname{Max}\{\lambda \geq 0: \lambda u \in K\} .
$$

If $\rho(K, \cdot)$ is positive and continuous, $K$ will be called a star body. Let $\mathcal{S}^{n}$ denote the set of star bodies in $\mathbb{R}^{n}$. Let $\tilde{\delta}$ denote the radial Hausdorff metric, that is defined as follows: if $K, L \in \mathcal{S}^{n}$, then $\tilde{\delta}(K, L)=\left|\rho_{K}-\rho_{L}\right|_{\infty}$ (see e.g. [3]).

If $K_{1}, \ldots, K_{r} \in \mathcal{S}^{n}$ and $\lambda_{1}, \ldots, \lambda_{r} \in \mathbb{R}$, then the radial Minkowski linear combination, $\lambda_{1} K_{1} \tilde{+} \cdots \tilde{+} \lambda_{r} K_{r}$, is defined by Lutwak (see [4]), as $\lambda_{1} K_{1} \tilde{+} \cdots \tilde{+} \lambda_{r} K_{r}=\left\{\lambda_{1} x_{1} \tilde{+} \cdots \tilde{+} \lambda_{r} x_{r}: x_{i} \in K_{i}\right\}$. Here, $\lambda_{1} x_{1} \tilde{+} \cdots \tilde{+} \lambda_{r} x_{r}$ equals $\lambda_{1} x_{1}+\ldots+\lambda_{r} x_{r}$ if $x 1, \ldots, x_{r}$ belong to a linear 1 -subspace of $\mathbb{R}^{n}$, and is 0 else. It has the following important property, for $K, L \in \mathcal{S}^{n}$ and $\lambda, \mu \geq 0$

$$
\rho(\lambda K \tilde{+} \mu L, \cdot)=\lambda \rho(K, \cdot)+\mu \rho(L, \cdot)
$$

For $K_{1}, \ldots, K_{r} \in \mathcal{S}^{n}$ and $\lambda_{1}, \ldots, \lambda_{r} \geq 0$, the volume of the radial Minkowski linear combination $\lambda_{1} K_{1} \tilde{+} \cdots \tilde{+} \lambda_{r} K_{r}$ is a homogeneous $n$ th-degree polynomial in the $\lambda_{i}$,

$$
V\left(\lambda_{1} K_{1} \tilde{+} \cdots \tilde{+} \lambda_{r} K_{r}\right)=\sum \tilde{V}_{i_{1}, \ldots, i_{n}} \lambda_{i_{1}} \cdots \lambda_{i_{n}}
$$

where the sum is taken over all $n$-tuples $\left(i_{1}, \ldots, i_{n}\right)$ whose entries are positive integers not exceeding $r$. If we require the coefficients of the polynomial in (2.2) to be symmetric in their argument, then they are uniquely determined. The coefficient $\tilde{V}_{i_{1}, \ldots, i_{n}}$ is positive and depends only on the star bodies $K_{i_{1}}, \ldots, K_{i_{n}}$. It is written as $\tilde{V}\left(K_{i_{1}}, \ldots, K_{i_{n}}\right)$ and is called the dual mixed volume of $K_{i_{1}}, \ldots, K_{i_{n}}$. If $K_{1}=\ldots=K_{n-i}=K, K_{n-i+1}=\ldots$ $=K_{n}=L$, the dual mixed volumes are written as $\tilde{V}_{i}(K, L)$. In particular, for $B$ the unit ball about $o, \tilde{V}_{i}(K, B)$ is written as $\tilde{W}_{i}(K)$ (see [5]).

For $K_{i} \in \mathcal{S}^{n}$, the dual mixed volumes were given by Lutwak (see [6]), as

$$
\tilde{V}\left(K_{1}, \ldots, K_{n}\right)=\frac{1}{n} \int_{S_{n-1}} \rho\left(K_{1}, u\right) \ldots \rho\left(K_{n}, u\right) \mathrm{d} S(u),
$$

For $K, L \in \mathcal{S}^{n}$ and $i \in \mathbb{R}$, the $i$ th dual mixed volume of $K$ and $L, \tilde{V}_{i}(K, L)$, is defined by,

$$
\tilde{V}_{i}(K, L)=\frac{1}{n} \int_{S^{n-1}} \rho(K, u)^{n-i} \rho(L, u)^{i} \mathrm{~d} S(u) .
$$


From (2.4), taking in consideration $\rho(B, u)=1$, if $K \in \mathcal{S}^{n}$, and $i \in \mathbb{R}$

$$
\tilde{W}_{i}(K)=\frac{1}{n} \int_{S^{n-1}} \rho(K, u)^{n-i} \mathrm{~d} S(u) .
$$

The well-known Brunn-Minkowski-type inequality for dual mixed volumes can be stated as follows [6]:

Theorem 2.1 Let $K, L \in \mathcal{S}^{n}$, and $i<n-1$. Then,

$$
\tilde{W}_{i}(K \tilde{+} L)^{1 /(n-i)} \leq \tilde{W}_{i}(K)^{1 /(n-i)}+\tilde{W}_{i}(L)^{1 /(n-i)},
$$

with equality if and only if $K$ and $L$ are dilates.

The inequality is reversed for $i>n-1$ and $i \neq n$.

In the following, we establish new Brunn-Minkowski-type inequalities for dual mixed volumes.

Theorem 2.2 Let $K, L \in \mathcal{S}^{n}$ and $i, j, k \in \mathbb{R}$.

(i) Let $i, j, k \in \mathbb{R}$ be different, such that $j, k<n-1$, and $(j-k) /(i-k)>1$. Then

$$
\begin{gathered}
\tilde{W}_{i}(K \tilde{+} L) \leq\left(\tilde{W}_{j}(K)^{1 /(n-j)}+\tilde{W}_{j}(L)^{1 /(n-j)}\right)^{(n-j)(k-i) /(k-j)} \\
\times\left(\tilde{W}_{k}(K)^{1 /(n-k)}+\tilde{W}_{k}(L)^{1 /(n-k)}\right)^{(n-k)(i-j) /(k-j)}
\end{gathered}
$$

with equality if and only if $K$ and $L$ are balls, or $1 /(n-i)=[1 /(n-j)+1 /(n-k)] / 2$, and $K$ and $L$ are dilates.

(ii) Let $i, j, k \in \mathbb{R}$ be different, such that $j, k>n-1$ and $j, k \neq n$, and $(j-k) /(i-k)<1$. Then

$$
\begin{gathered}
\tilde{W}_{i}(K \tilde{+} L) \geq\left(\tilde{W}_{j}(K)^{1 /(n-j)}+\tilde{W}_{j}(L)^{1 /(n-j)}\right)^{(n-j)(k-i) /(k-j)} \\
\left(\tilde{W}_{k}(K)^{1 /(n-k)}+\tilde{W}_{k}(L)^{1 /(n-k)}\right)^{(n-k)(i-j) /(k-j)}
\end{gathered}
$$

with equality if and only if $K$ and $L$ are balls, or $1 /(n-i)=[1 /(n-j)+1 /(n-k)] / 2$, and $K$ and $L$ are dilates..

Proof We begin with the proof of (i). From (2.1), (2.5) and (1.3), we have

$$
\begin{aligned}
& \tilde{W}_{i}(K \tilde{+} L)=\frac{1}{n} \int_{S^{n-1}} \rho(K \tilde{+} L, u)^{n-i} \mathrm{~d} S(u)=\frac{1}{n} \int_{S^{n-1}}(\rho(K, u)+\rho(L, u))^{n-i} \mathrm{~d} S(u) \\
& \leq \frac{1}{n}\left[\left(\int \rho(K, u)^{n-j} \mathrm{~d} x\right)^{1 /(n-j)}+\left(\int \rho(L, u)^{n-j} \mathrm{~d} x\right)^{1 /(n-j)}\right]^{(n-j)(k-i) /(k-j)} \\
& \times\left[\left(\int \rho(K, u)^{n-k} \mathrm{~d} x\right)^{1 /(n-k)}+\left(\int \rho(L, u)^{n-k} \mathrm{~d} x\right)^{1 /(n-k)}\right]^{(n-k)(i-j) /(k-j)} \\
& =\left(\tilde{W}_{j}(K)^{1 /(n-j)}+\tilde{W}_{j}(L)^{1 /(n-j)}\right)^{(n-j)(k-i) /(k-j)}\left(\tilde{W}_{k}(K)^{1 /(n-k)}+\tilde{W}_{k}(L)^{1 /(n-k)}\right)^{(n-k)(i-j) /(k-j)},
\end{aligned}
$$

with equality if and only if as stated in (i).

Similarly, case (ii) of Theorem 2.2 easily follows.

Remark 2.3 For (i) of Theorem 2.2, for $n-i>1$, letting $s=n-i+\varepsilon, t=n-i-\varepsilon$, when $i, j, k$ are different, $n-j, n-k>1$, and $(k-j) /(k-i)=2>1$, and letting $\varepsilon \rightarrow 0$, we get the following result: Let $K, L \in \mathcal{S}^{n}$, and $i<n-1$. Then, 


$$
\tilde{W}_{i}(K \tilde{+} L)^{1 /(n-i)} \leq \tilde{W}_{i}(K)^{1 /(n-i)}+\tilde{W}_{i}(L)^{1 /(n-i)},
$$

with equality if and only if $K$ and $L$ are dilates.

This is just the well-known inequality (2.6) in Theorem 2.1.

For (ii) of Theorem 2.2, for $n-i<1$ and $n-i \neq 0, s=n-i+\varepsilon, t=n-i+2 \varepsilon$, when $i$, $j, k$ are different, $n-j, n-k<1$ and $n-j, n-k \neq 0$, and $(k-j) /(k-i)=1 / 2<1$, and letting $\varepsilon \rightarrow 0$, we get the following result:

Let $K, L \in \mathcal{S}^{n}$, and $i<n-1$ and $i \neq n$. Then,

$$
\tilde{W}_{i}(K \tilde{+} L)^{1 /(n-i)} \geq \tilde{W}_{i}(K)^{1 /(n-i)}+\tilde{W}_{i}(L)^{1 /(n-i)},
$$

with equality if and only if $K$ and $L$ are dilates.

This is just an reversed form of inequality (2.6).

\section{Acknowledgements}

The authors wish to thank the referee for his many excellent suggestions for improving the original manuscript. This Research is supported by National Natural Science Foundation of China(10971205) and in part by a HKU URC grant.

\section{Author details}

${ }^{1}$ Department of Mathematics, China Jiliang University, Hangzhou 310018, China 2Department of Mathematics, The University of Hong Kong, Pokfulam Road, Hong Kong

\section{Authors' contributions}

C-JZ and W-SC jointly contributed to the main results Theorems 1.2 and 2.2, Both authors read and approved the final manuscript.

\section{Competing interests}

The authors declare that they have no competing interests.

Received: 23 February 2011 Accepted: 27 September 2011 Published: 27 September 2011

\section{References}

1. Beckenbach, EF, Bellman, R: Inequalities. Springer, Berlin-Göttingen (1961)

Hardy, GH, Littlewood, JE, Pólya, G: Inequalities. Cambridge University Press, Cambridge (1934)

Schneider, R: Convex Bodies: The Brunn-Minkowski Theory. Cambridge University Press, Cambridge (1993)

Lutwak, E: Intersection bodies and dual mixed volumes. Adv Math. 71, 232-261 (1988). doi:10.1016/0001-8708(88)90077-1

Gardner, RJ: Geometric Tomography. Cambridge University Press, New York (1996)

Lutwak, E: Dual mixed volumes. Pacific J Math. 58, 531-538 (1975)

Cite this article as: Zhao and Cheung: On Minkowski's inequality and its application. Journal of Inequalities and Applications 2011 2011:71.

\section{Submit your manuscript to a SpringerOpen ${ }^{\circ}$} journal and benefit from:

- Convenient online submission

- Rigorous peer review

- Immediate publication on acceptance

- Open access: articles freely available online

- High visibility within the field

- Retaining the copyright to your article

Submit your next manuscript at $>$ springeropen.com 